\title{
Data-Driven Diagnostics and Prognostics for Modelling the State of Health of Maritime Battery Systems - a Review
}

\author{
Erik Vanem ${ }^{1,2}$, Øystein Åsheim Alnes ${ }^{1}$, and James Lam ${ }^{3}$ \\ ${ }^{1}$ DNV Group Research \& Development, Hфvik, Norway \\ Erik.Vanem@dnv.com \\ Oystein.Alnes@dnv.com \\ ${ }^{2}$ Department of Mathematics, University of Oslo, Oslo, Norway \\ erikvan@math.uio.no \\ ${ }^{3}$ DNV Energy, London, UK \\ James.Lam@dnv.com
}

\begin{abstract}
Battery systems are becoming an increasingly attractive alternative for powering ocean going ships, and the number of fully electric or hybrid ships relying on battery power for propulsion and maneuvering is growing. In order to ensure the safety of such ships, it is of paramount importance to monitor the available energy that can be stored in the batteries, and classification societies typically require that the state of health of the batteries can be verified by independent tests - annual capacity tests. This paper discusses data-driven diagnostics for state of health modelling for maritime battery systems based on operational sensor data as an alternative approach. It presents a comprehensive review of different datadriven approaches to state of health modelling, and aims at giving an overview of current state of the art. Furthermore, the various methods for data-driven diagnostics are categorized in a few overall approaches with quite different properties and requirements with respect to data for training and from the operational phase. More than 300 papers have been reviewed, many of which are referred to in this paper. Moreover, some reflections and discussions on what types of approaches can be suitable for modelling and independent verification of state of health for maritime battery systems are presented.
\end{abstract}

\section{INTRODUCTION}

There is currently a significant push for emission reduction and a change to more environmentally friendly technologies for maritime transport. Electric or hybrid ships using batteries

\footnotetext{
Erik Vanem et al. This is an open-access article distributed under the terms of the Creative Commons Attribution 3.0 United States License, which permits unrestricted use, distribution, and reproduction in any medium, provided the original author and source are credited.
}

is an attractive alternative for many shipping segments with significant environmental benefits and large potential for fuel, cost and emission savings.

The safety of battery-powered ships is important. Fire and explosion are obvious risks, but another central aspect is ensuring that the available energy stored in the batteries is sufficient to cover the power demand. Loss of propulsion power in a critical situation can lead to serious accidents such as collision or grounding. Therefore, a reliable estimation and prediction of the actual available energy of a battery is crucial.

Battery systems are ageing, meaning that the energy storage capacity degrades by calendar time and by charge/discharge cycles. The ageing process affects both the amount of charge that can be stored and the performance of the power delivery. For ships relying on energy from onboard battery systems, it is important to ensure that the capacity of the battery system is sufficient for the safe operation of the vessel at all times. Thus, accurate evaluation and verification of the capacity and performance of maritime battery systems is crucial to safe and sustainable operation of battery powered ships. It is noted that other aspects of battery degradation may be equally important. For example, degradation does not only affect the capacity, but also fire safety and thermal runaway properties are influenced by degradation (Geisbauer, Wöhrl, Mittmann, \& Schweiger, 2020; D. Ren et al., 2019).

Due to its safety criticality, class societies typically require annual validation testing of battery State of Health $(\mathrm{SOH})$ for ships utilizing battery systems for propulsion or manoeuvring purposes. There are many challenges with this approach, and data-driven approaches to $\mathrm{SOH}$ monitoring and prediction are believed to be attractive alternatives. From a practical point 
of view, the annual capacity test is time consuming and typically requires that the ship is taken out of operation for one full day per year. Moreover, the accuracy of the test is questionable due to several factors influencing the results, such as variability in loads, temperatures and Depth of Discharge (DOD). Maritime battery systems are typically designed for a 10-year lifetime while the ships are designed for 25-30 years. When battery systems are approaching their end of useful life (EOL) reliable estimation of $\mathrm{SOH}$ will become much more important and making correct decisions on remaining useful life (RUL) will have great financial and safety implications.

This paper aims at describing the state of the art in data-driven methods for $\mathrm{SOH}$ estimation of maritime battery systems. It is based on a thorough literature survey, presented in (Vanem, Bertinelli Salucci, Bakdi, \& Alnes, 2021), and outlines various approaches reported in the scientific and engineering literature for utilizing sensor data to estimate the effect of degradation on the available capacity of such battery systems.

\subsection{Condition Monitoring for Battery Systems}

With a rechargeable battery system, the amount of energy available at all times will vary continuously as the battery is repeatedly charged and discharged, and the state of charge (SOC) is a measure of the extent to which the battery is charged relative to its capacity. That is, a fully charged battery will have SOC $=100 \%$ and a fully discharged battery will have $\mathrm{SOC}=0 \%$. The State of Latent Energy (SoLE) is a a similar measure of the amount of available useful energy in the battery (Rozas, Troncoso-Kurtovic, Ley, \& Orchard, 2021) that do not rely on a normalization constant.

The capacity of a battery to store energy will typically degrade over time, and the state of health $(\mathrm{SOH})$ is a measure of the battery's capacity relative to its nominal capacity, that is, the initial capacity when the battery is new. Formally, the State of Health of a battery can be defined as

$$
S O H=\frac{C_{\text {Available }}}{C_{\text {Nominal }}} \times 100 \%,
$$

where $C_{\text {Available }}$ denotes the available capacity of the battery and $C_{\text {Nominal }}$ refers to the nominal capacity. It should be noted that alternative definitions of SOH exist, for example based on internal resistance, and that these are generally not identical. Although there is a correlation between capacitybased $\mathrm{SOH}$ and resistance-based $\mathrm{SOH}$ it is important to be aware that $\mathrm{SOH}$ is not unambiguously defined. In this paper, the main focus is on available capacity and unless otherwise noted, $\mathrm{SOH}$ should be taken to mean capacity-based state of health as defined in Eq. (1).

The cycle life or battery life of a rechargeable battery refers to the number of full discharge-charge cycles the battery can experience before its end of life, and many different factors influence the actual cycle life, including the rate and depth of the cycles and temperature. Alternatively, battery life can sometimes be described in terms of cumulative discharge (total amount of charge delivered by the battery over its lifetime) or equivalent full cycles (summation of partial cycles as fractions of full charge-discharge cycles).

Currently, all maritime battery suppliers are required to have a $\mathrm{SOH}$ estimation algorithm and to verify the $\mathrm{SOH}$ annually through in-situ capacity testing. As ship-to-shore connectivity has immensely improved over the past few years it is natural to evaluate whether a sensor-based monitoring system can both reduce downtime for the operator and improve the quality of the $\mathrm{SOH}$ verification.

Condition monitoring systems typically include diagnostics and prognostics. Within such a framework, state of health estimation corresponds to the diagnostics part where reliable estimation of state of health reflects the energy storage capacity of the battery at any given time. This would be influenced by the operating history of the battery system. Prognostics in this context would amount to predicting the remaining useful life of the battery or the time until the battery needs to be replaced. This would require some threshold to be specified for when the battery reaches its end of life, which could be in terms of SOH below a specified limit, as well as some assumptions on the future operation of the system and should predict future degradation trends based on this.

\subsection{Battery Technologies and Terminology}

Batteries have been around for a long time and battery technologies are continuously being developed since the first electrochemical batteries were invented in the late 18th century. Fundamentally, an electrochemical battery cell must consist of an inner ionic channel allowing for transport of ions, two materials with interfaces where the exchange of electrons and chemical reactions can occur and an outer electrical channel for transport of electrons. The materials where exchange of electrons occur are referred to as the positive and negative electrodes and the ionic channel are referred to as the electrolyte. Currently, the most widespread type of rechargeable batteries are lithium-ion batteries, and there exist a range of different chemistries with different characteristic. An overview of maritime and offshore battery systems can be found in (DNV GL, 2016).

For the purpose of establishing data-driven models to estimate state of health and predict remaining useful life, it should be acknowledged that the different chemistries may have very different characteristics with regards to how different factors influence the degradation. Furthermore, battery cells can be of different forms and types, and the most common lithium-ion cell types for maritime applications are cylindrical, prismatic and soft pouch cells. These form factors have different performance characteristics. 
The C-rate is a measure of the rate of which a battery is being charged or discharged and is defined as the current through the battery divided by the current needed to discharge the battery's nominal rated capacity in one hour. It has the unit $1 / h$ (per hour).

A battery consist of one or more connected electrochemical cells. Cells can be connected in series in order to increase the electric potential of the battery or connected in parallel in order to increase the capacity of the battery. More complicated configurations and architectures may involve several cells in different combinations of series and parallel connections. There may be uneven loads and temperatures on the different cells, and different cells within the same battery may experience different degradation trends. Often, lab testing is carried out on single cells and operational sensor data from a battery system typically include measurement both at cell and system level.

A rechargeable battery will be operated in cycles consisting of charging, discharging and rest periods throughout its operational life. The charging and discharging cycles could be at different rates (C-rates) and depths (range of SOC), and the rest periods can occur at different state of charge. All of these are influencing the degradation of the battery. During normal operation the batteries will often be operated under variable conditions, with constantly varying rates and depths of the cycles and rest periods, and sensor measurements of battery voltage, current and temperature over time can describe the operational profile

Modern batteries are equipped with a battery management system (BMS), which are very important for the safe operation of the battery, and also for optimizing the use of the battery (Weicker, 2014). A BMS should monitor the state of a battery at all times and protect the battery from operating outside its safe operating area to prevent accidents such as explosion or thermal runaway. It collects sensor measurements of basic parameters such as voltage, current and temperature and uses these to calculate and monitor various derived parameters and quantities such as state of charge and state of health.

Some factors that influence the degradation of a battery are well known, even though the degradation mechanisms may be different for different battery types and chemistries. The degradation leads to loss of capacity, power fade and increase in internal resistance. As a result of this, the terminal voltage and range of state of charge will be reduced as the battery degrades. Typically, degradation and capacity loss are ascribed to calendar ageing and cyclic ageing effects. A list of factors influencing battery health presented in (Balagopal \& Chow, 2015) include temperature, charging and discharging cycles, depth of discharge, overcharging charge/discharge rate and calendar ageing. An overview of important battery degradation mechanisms as well as their causes and effects are also given in e.g. (Vetter et al., 2005; Birkl, Roberts, McTurk, Bruce, \& Howey, 2017).

The degradation may not be similar in the beginning of life (BOL) and when approaching end of life (EOL). Typically, one expect to observe a so-called knee-point in the degradation curves, where a sudden change from relatively moderate degradation to a more aggressive degradation occurs towards the EOL. Maritime batteries should typically be replaced before a knee-point occurs in the degradation curves, to avoid swiftly deteriorating battery capacities during operation.

The availability of high quality data with sufficient accuracy, resolution, relevance and completeness is important for developing and training data-driven models for state of health estimation. There are essentially two different ways of obtaining such data, i.e. data collected from measurements of battery systems during operation and data collected from laboratory experiments. Some limitations of actual measurements are that the availability of sufficiently long time series are scarce and the fact that one may have only partial information and control of the conditions under which the data is collected. Batteries are also typically replaced before their end of life, so data from the critical period when the batteries approach their end of useful life will often not be available. On the other hand, lab data can be collected under controlled, often idealized conditions, where for example temperatures and loads can be kept constant throughout the experiments. However, this may not be very representative for realistic operation of the batteries, with variable loads and operating conditions.

\subsection{Classification Rules for Electric Ships}

Electrical power systems have been used onboard ships for a long time, and recently fully electrical or hybrid ships depending on battery power for propulsion have become attractive for many ship segments. Ocean going ship are subject to classification rules (DNV GL, 2020a), and DNV has an additional class notation, BATTERY, for battery powered vessels (DNV GL, 2020b). The Battery(Power) class notation is required for all ships - all-electric or hybrid - that relies on battery power for propulsion and the Battery(Safety) notation applies to all vessels with lithium-ion battery systems with an aggregated rated capacity of more than $20 \mathrm{kWh}$ and not having the Battery(Power) notation. The annual capacity test is a requirement of the Battery(Power) notation.

\section{LITERATURE SURVEY ON DATA-DRIVEN MODELS FOR SOH ESTIMATION}

This paper aims at presenting state-of-the-art in data-driven models for state of health of maritime battery systems. The amount of literature on this topic is enormous and it seems an impossible task to cover all relevant papers and reports in the academic and engineering literature in detail. Notwithstanding, the literature survey presented herein are believed to give 
a fair overview of different approaches to data-driven modelling of the condition of batteries, with an emphasis on the more recent literature and on the various overall approaches that can be taken.

In the following, a high-level review of methods proposed in the scientific literature will be given, with a focus on datadriven methods based on sensor measurements from batteries in operation. An effort is made to group models in a few main categories, although some proposals may include elements from various categories. Typically, methods are grouped into experimental methods such as various forms of measurements, model-based methods relying on electrochemical or equivalent circuit models and pure data-driven methods. However, the distinction is not always crisp, and a combination of techniques will typically be employed.

\subsection{Previous review papers}

Some recent review papers on ageing mechanisms have been presented in e.g. (Barré, Deguilhem, Grolleau, \& Gérard, 2013; Kabir \& Demirocak, 2017; Han, Lu, et al., 2019; Saqli, Bouchareb, Oudghiri, \& M'Sirdi, 2020). A range of methods for describing battery degradation are summarized in e.g. (Barré et al., 2013), which concludes that models that meet all performance criteria do not yet exist. Other review papers generally concur to this conclusion and reliable data-driven approaches for estimating battery capacity remains a challenge. See e.g. (Pelletier, Jabali, Laporte, \& Veneroni, 2017; Farmann, Waag, Marongiu, \& Sauer, 2015; Pastor-Fernández, Yu, Widanage, \& Marco, 2019; Balagopal \& Chow, 2015; Cuma \& Koroglu, 2015; Berecibar, Gandiaga, et al., 2016; Ungurean, Cârstoiu, Micea, \& Groza, 2017; Huixin, Qin, Li, \& Zhao, 2020; Xiong, Li, \& Tian, 2018; Lipu et al., 2018; Lucu, Martinez-Laserna, Gandiaga, \& Camblong, 2018) for recent reviews on capacity estimation. Several review studies focus on prognostics and RUL estimation of lithium-ion batteries, see e.g. (Y. Li et al., 2019; L. Wu, Fu, \& Guan, 2016; Su \& Chen, 2017; Saha, Goebel, \& Chrisophersen, 2009).

\subsection{Direct Measurement Techniques}

Different approaches for more or less direct measurements of state of health exist and are proposed for online SOH estimation. Some of these can be based on continuous measurements such as time series of currents, voltages and temperatures, whereas others are based on measurements collected during particular experiments or procedures (Karlsen, Dong, Yang, \& Carvalho, 2019). For example, the annual test currently required for maritime battery systems used for propulsion utilizes a coulomb counting technique and a controlled charging/discharging procedure. This is one approach to $\mathrm{SOH}$ verification, but the need for specific charging and discharging cycles under controlled environments, with con- stant temperature and C-rate, means that normal operations need to be disrupted for a period of time. Other measurement techniques also exist, see e.g. a more comprehensive overview in (Barai et al., 2019). Ideally, methods that can be used based on continuous measurements of variables that are routinely collected under normal operations without the need for specific instrumentation or procedures are preferable.

\subsubsection{Coulomb Counting}

Coulomb counting, also referred to as current integration method, integrates the current to or from the battery during a full cycle to determine the capacity directly, according to the basic relation

$$
Q=\int_{t_{0}}^{t_{1}} I(\tau) d \tau
$$

where $Q$ is the capacity, $I(t)$ is the current at time $t$ and $t_{0}$ and $t_{1}$ refers to the times of SOC $=0 \%$ and SOC $=100 \%$, respectively. That is, the current is integrated over a full cycle from full to empty (or from empty to full) to count how much electric charge the battery can store. Often, the equation above can be modified by also including the Coulombic efficiency, which is tacitly assumed to be unity in Eq. (2). One practical problem with this approach is that it requires a full charge/discharge cycle to be able to estimate the maximum capacity and this is hardly ever experienced in actual normal operations. Moreover, the measurements need to be performed under controlled conditions, with constant, typically low, C-rate and a specific ambient temperature and is therefore not directly applicable as an online method. In addition, subjecting the battery to full cycles between $0 \%$ and $100 \%$ may contribute to accelerated degradation and such tests risk shortening the lifetime of the battery. The annual test for maritime battery systems are based on Coulomb counting and therefore needs to take the vessel out of service to perform a series of controlled charge and discharge cycles.

Capacity estimation can possibly be based on coulomb counting of deep cycles (not necessarily full), at reasonably homogeneous conditions with respect to $\mathrm{C}$-rates and temperatures. The relationship between total capacity, $Q$ and state of charge at times, $t_{1}$ and $t_{2}$ is as follows, where also the Coulombic efficiency $\eta$, is included.

$$
\Delta S O C=S O C\left(t_{2}\right)-\operatorname{SOC}\left(t_{1}\right)=\frac{1}{Q} \int_{t_{1}}^{t_{2}} \eta I(\tau) d \tau
$$

Note, however, that for this approach to be useful there is a need for accurate and reliable SOC estimates.

Estimation of SOH based on Coulomb counting of partial cycles, is proposed in (Stroe, Knap, \& Schaltz, 2018; Q. Yang et al., 2017), indicating that the reduced voltage range measurements are likely to underestimate the capacity fade, see also (Meng et al., 2019). Coulomb counting are also often pro- 
posed to be used together with other data-driven or modelbased techniques. It is possible to include a current correction term in the Coulomb counting procedure to account for the fact that capacity generally decreases as discharge current (C-rate) increases (Z. Deng, Yang, Cai, \& Deng, 2017). The Peukert equation describes the relationship between the discharge current $(I)$ and the discharge time $(t)$ by stating that $I^{k} t$ is a constant, where $k$ is the Peukert coefficient (Doerffel \& Sharkh, 2006; Z. Deng, Yang, Cai, Deng, \& Sun, 2016). However, this requires the battery to be discharged at a constant C-rate throughout the cycle (Doerffel \& Sharkh, 2006), and also at constant temperature. Extensions of the Coulomb counting method are discussed in (Gismero, Schaltz, \& Stroe, 2020)

\subsubsection{Hybrid Pulse Power Characterisation (HPPC) and Electrochemical Impedance Spectroscopy (EIS)}

HPPC and EIS are methods to measure the electrochemical response of certain inputs. HHPC measures the cell voltage response to short high-current charge/discharge pulses and EIS measures the frequency response of the battery by measuring the impedance over a range of $\mathrm{AC}$ input at different frequencies. It yields a impedance spectrum from which it is possible to estimate various battery characteristics, such as charge transfer resistance, capacitance and ohmic resistance as different frequencies are associated with different mechanisms in the battery, and to relate this to state of health (Blanke et al., 2005; Tröltzsch, Kanoun, \& Tränkler, 2006; Pérez, Benavides, Rozas, Seria, \& Orchard, 2018). A passive impedance measurement technique is proposed in (Bohlen, 2008) to alleviate the need for specific hardware implementations, allowing the impedance spectrum to be estimated from arbitrary excitation signals by way of digital filters. See also (Howey, Mitcheson, Yufit, Offer, \& Brandon, 2014) for an example of online EIS measurements, and . An extension of the EIS to study also higher order harmonics and nonlinear responses is proposed in (Harting, Wolff, Röder, \& Krewer, 2017; Harting, Schenkendorf, Wolff, \& Krewer, 2018).

EIS measurements are used together with model-based approaches in (Kuipers et al., 2020; X. Wang, Wei, \& Dai, 2019) and with data-driven approaches in e.g. (Y. Zhang et al., 2020) for SOH estimation and RUL prediction.

\subsubsection{Incremental Capacity Analysis (ICA) and Differ- ential Voltage Analysis (DVA)}

IVA and DVA measure the change in charge (Q) and voltages (V) during charging/discharging and estimates the gradient curves, $d Q / d V$ and $d V / d Q$, respectively, to determine changes in electrochemical properties. Such curves will typically exhibit features like plateaus and peaks that can be associated with different mechanisms and phases in the battery and changes in these features can be ascribed to battery degra- dation. It is also possible to apply this method for partial charging curves, which is a huge advantage for online monitoring. However, two major challenges with this approach for online monitoring based on real-time sensor data is that a constant and low current is typically needed in order to acquire accurate curves and the differentiation of noisy, discrete data to obtain the IC $(d Q / d V)$ and DV $(d V / d Q)$ curves (Feng et al., 2020). An example of a charge-voltage curve and the corresponding IC $(d Q / d V)$ curve is shown in Figure 1, illustrating that flat parts of the charge-voltage curve appears as peaks in the $d Q / d V$ curve.
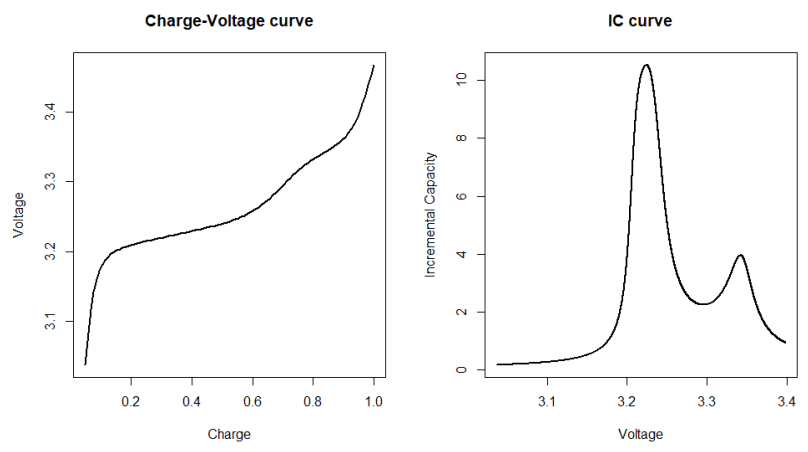

Figure 1. A simple example of an incremental capacity curve. Plateaus in the charge-voltage curve correspond to peaks in the IC curve

Such curves may be estimated in different ways, including curve fitting, parametric models and machine learning methods (Han, Feng, et al., 2019; Weng, Sun, \& Peng, 2013; Y. Li, Abdel-Monem, et al., 2018; He, Wei, Bian, \& Yan, 2020). Various smoothing techniques can also be applied to obtain smooth curves from noisy measurements (Jiang, Dai, \& Wei, 2020). Having established the curves, various ways of extracting features can be tried in order to regress capacity degradation on the selected features (X. Li, Wang, Zhang, Zou, \& Dorell, 2019; Jiang et al., 2020; Tang et al., 2018; C. Lin, Cabrera, Yu, Yang, \& Tsui, 2020; Riviere, Sari, Venet, Meniere, \& Bultel, 2019; L. Zheng, Zhu, Lu, Wang, \& He, 2018), e.g. using support vector regression (Weng, Cui, Sun, \& Peng, 2013), linear models (Jiang et al., 2020). A current interrupt technique is introduced to evaluate the cell resistance in order to account for the effect of different C-rates in ICA in (Fly \& Chen, 2020).

A somewhat similar method based on charge and discharge data estimates the probability density function of voltages during a discharge cycle by way of kernel density fitting of discrete voltage measurements (Feng et al., 2013). This method is referred to as the pdf-method and is a simplified variant of ICA where the need to fit a curve to the charge/discharge data are eliminated. Similarly as with ICA, the probability density function will exhibit clear peaks around voltage plateaus, that is, voltages that occur more fre- 
quently during a charge or discharge cycle, and the idea is that the state of the battery can be inferred by these peaks.A fusion of Coulomb counting and differential voltage analysis is proposed in (S. Zhang, Guo, Dou, \& Zhang, 2020) as a model-free approach to obtain $\mathrm{SOH}$ estimation from constant current discharge data.

\subsubsection{Other Direct Measurement Techniques}

A differential thermal voltammetry approach is proposed in (B. Wu et al., 2015), where voltage and temperature measurements in galvanostatic operations are used to model state of health. This allows shorter measurement time than slow rate cyclic voltammetry analysis (Stiaszny, Ziegler, Krauß, Schmidt, \& Ivers-Tiffée, 2014; Stiaszny, Ziegler, Krauß, Zhang, \& Ivers-Tiffée, 2014). A differential heat analysis based on measuring gradient heat flux and temperature after discharge is proposed for SOH estimation in (Murashko et al., 2019). State of health estimation based on the Ampere-hour throughput - voltage curve and fitting a parametric curve to these is proposed in (Le \& Tang, 2011).

\subsection{State-Space Models with Observers}

A different approach to battery modelling relies on models that approximate the battery dynamics. Typically, these may be referred to as state-space models where sensor data can be used to estimate model parameters corresponding to underlying unobservable states using so-called observers such as various variants of the Kalman filter or particle filters. Two main classes of such models are equivalent circuit models and electrochemical models. Such models may also be combined with experimental methods or direct measurements and data-driven methods to estimate state parameters and state of health. Pure data-driven models can also be established, such as the local model network (LMN) presented in (Hametner, Jakubek, \& Prochazka, 2018), where a set of local regression models are used to establish a non-linear battery model. A Brownian motion model with drift was proposed in (Dong, Yang, Wei, Wei, \& Tsui, 2020) to model battery degradation as a hidden state model based on observed health indices (that could be capacity loss or resistance increase).

Equivalent circuit models (ECM) describe the voltage-current characteristics of a battery by a model of an electrical circuit with different elements such as resistors and capacitors in different series- and parallel configurations. Having established a ECM for the battery, the state of the battery is described by the battery model parameters. These are typically unobserved, but may be estimated based on measurements using various optimization techniques such as different variants of least squares methods. Various forms of constrained and regularized optimization may be employed to avoid unreasonable parameter estimates (Tian, Wang, Chen, \& Fang, 2020) and forgetting factors can be used to avoid saturation problems by giving less weight to previous data compared to more recent ones (L. Chen, Lü, Lin, Li, \& Pan, 2018). Model parameters are typically changing dynamically over time and observers such as Kalman filter and particle filters can be used to dynamically update model parameters and unobserved model states. Extensions of the Kalman filter to handle non-linear state transition and observation models include the extended Kalman filter and the unscented Kalman filter (see e.g. (Plett, 2004a, 2004b, 2004c)). The effect of temperature may be included in such models by coupling the ECM with an energy balance or thermal model, see e.g. (Karlsen et al., 2019; Bian, Liu, Yan, Zou, \& Zhao, 2020). An ECM is used in (Ley \& Orchard, 2021) to adapt a statespace model to learn a polarising impedance surface used for capacity degradation modelling.

Electrochemical models typically consist of a simplified set of electrochemical equations that model the transport of charge between the positive and negative electrode in the battery cells based on the underlying physics. They describe the charge flows through the electrolyte and voltage drops at the cathode, anode and separator of the battery cells and typically include a set of differential equations, several model parameters, model states and some measurable model output. The model parameters are typically identified from battery dimensions and chemistry or are estimated based on data. Examples of such electrochemical models are given in (Bole, Kulkarni, \& Daigle, 2014; Daigle \& Kulkarni, 2013; Bi, Yin, \& Choe, 2020; C. Lin, Xing, \& Tang, 2017). Battery ageing and degradation can be modelled by changes in model parameters describing e.g. the internal resistance and charge capacity of the battery.

\subsection{Regression Type Models}

Regression model range from simple linear regression models assuming a linear relationship between a set of explanatory variables and a response variable to complex machinelearning (ML) type of regression models for more complicated and non-linear relationships. One advantage of complicated models is that more accurate models may be constructed when accounting for non-linearities. However, a parsimonious model can also be preferred as it will be less likely to overfit training data and be more easily interpreted. In general, in order to use regression type models there is a need for representative training data so that the model can learn the relationship between the input variables and the response. For batteries, this means that battery test data is needed, where both the explanatory variables and the response is measured, typically based on laboratory tests. However, it is uncertain how representative the typical lab test data are for the degradation caused by more random duty cycles experienced in the field.

Simple linear regression models are proposed in (D. Wang, 
Kong, Yang, Zhao, \& Tsui, 2020; Huang, Tseng, Liang, Chang, \& Pecht, 2017; Severson et al., 2019; Tang et al., 2018), and different regression models for $\mathrm{SOH}$ based on polynomial functions of cycle number as the only variable and polynomial and exponential functions of fully discharged voltage and internal resistance are compared in (Tseng, Liang, Chang, \& Huang, 2015). A kernel ridge regression model is suggested for $\mathrm{SOH}$ estimation in (Y. Li, Sheng, Cheng, Stroe, \& Teodorescu, 2020). The relationship between capacity, accumulated charge and ranges of state of charge during cycling expressed in Eq. (3) is formulated as a regression problem in (Plett, 2011), where the total capacity is a regression coefficient between measured changes in state of charge (predictor) and accumulated charge obtained by Coulomb counting (response). A similar approach framing maximum capacity estimation as a total least square problem is taken in (T. Kim et al., 2015), where a Rayleigh quotientbased algorithm is employed to estimate capacity recursively.

Three data-driven methods for $\mathrm{SOH}$ estimation are compared in (Berecibar, Devriendt, et al., 2016), i.e. a linear regression model based on ordinary least squares, a multilayer perceptron neural network and a support vector machine. It is emphasized that the linear model is more comprehensible but that the neural network provides slightly more robust results. Machine learning regression models proposed in the literature include support vector regression (Z. Deng et al., 2017; D. Yang, Wang, Pan, Chen, \& Chen, 2018; Klass, Behm, \& Lindberg, 2014; Weng, Cui, et al., 2013; X. Li, Yuan, \& Wang, 2020b; Z. Wang, Zeng, Guo, \& Qin, 2018; Nuhic, Terzmehic, Soczka-Guth, Buchholz, \& Dietmayer, 2013; Nuhic, Bergdolt, Spier, Buchholz, \& Dietmayer, 2018; Shu, Li, Zhang, et al., 2020; Y. Deng et al., 2019; Shu, Li, Shen, et al., 2020; Zhao, Qin, Zhao, \& Feng, 2018; Harting et al., 2018; W. Pan, Chen, Zhu, Tang, \& Wang, 2020), relevant vector machines (Guo, Cheng, \& Yang, 2019; Nuhic et al., 2018), different variants of artificial neural networks (ANN) (You, Park, \& Oh, 2016; Y. Zhang, Xiong, He, Qu, \& Pecht, 2019a; S. Zhang et al., 2019; Naha et al., 2020; Sbarufatti, Corbetta, Giglio, \& Cadini, 2017), extreme learning machines (H. Pan, Lü, Wang, Wei, \& Chen, 2018; Y. Ma, Wu, Guan, \& Peng, 2020; T. Xu, Peng, \& Wu, 2021), recurrent neural networks (Kwon et al., 2020; P. Li et al., 2020; Ungurean, Micea, \& Cârstoiu, 2020), probabilistic neural networks (H.-T. Lin, Liang, \& Chen, 2013), deep learning (Khumprom \& Yodo, 2019; Fan, Xiao, Li, Yang, \& Tang, 2020; S. Shen, Sadoughi, Chen, Hong, \& Hu, 2019; W. Li et al., 2021), Gaussian processes regression (Richardson, Birkl, Osborne, \& Howey, 2019; Richardson, Osborne, \& Howey, 2019; Khaleghi, Firouz, Van Mierlo, \& Van den Bossche, 2019; Lucu et al., 2020b; X. Li, Yuan, \& Wang, 2020a) and random forests (Z. Chen, Sun, Shu, Shen, \& Xiao, 2018; Y. Li, Zou, et al., 2018; H. Xu, Peng, \& Su, 2018; Lemprecht, Riesterer, \& Steinhorst, 2020; Song, Fei, \& Xia, 2020).
The capacity of batteries is not measured directly by sensors and are therefore not available for each cycle in online battery data. If available at all, capacities will only be available for limited cycles. This raises the need for semi-supervised learning, as addressed in (Yu, Yang, Wu, Tang, \& Dai, 2020). In order to address the problem of insufficient training data, concepts of transfer learning and ensemble learning are incorporated in (S. Shen, Sadoughi, Li, Wang, \& Hu, 2020).

\subsection{Time-Series Models}

Time-series models represent a different approach to modelling capacity fade. Rather than estimating capacity and state of health by regressing on some explanatory variables, timeseries models estimate capacity based on previous observed capacities and model the serial dependence in observed capacities. Hence, based on a history of capacity measurements, current and future capacity values can be estimated. Typically, time-series models can be used for forecasting and predicting remaining useful life of batteries. Different types of classical time-series models are proposed in the literature for modelling battery capacity, including nonlinear autoregressive (AR) models (Liu et al., 2014), AR model with covariates in multiple-change-point linear model (C.-P. Lin et al., 2020) and autoregressive integrated moving average (ARIMA) models (Zhou \& Huang, 2016) and models based on Wiener processes (X. Xu et al., 2019; D. Shen, Wu, Kang, Guan, \& Peng, 2021).

Time series based on more advanced ML models have also been proposed, e.g. based on recurrent neural networks (X. Li, Zhang, Wang, \& Dong, 19; G. Ma et al., 2019; Ungurean et al., 2020), and Gaussian processes (L. Li, Wang, Chao, Zhou, \& Xie, 2016; X. Li et al., 2020a; X. Li, Wang, \& Yan, 2019).

Notwithstanding several approaches where time-series models have been used for $\mathrm{SOH}$ prediction, such models generally project future values based on historical observations of the capacity, rather than regressing capacity on other explanatory variables. Hence, such models are believed to be more relevant for prognostics applications than for diagnostics and such methods are deemed less relevant for estimating SOH of maritime battery systems based on sensor measurements.

\subsection{Survival Type Models}

Survival and event history modelling is a separate branch of statistics that are used to model time-to-event data. If for example a battery's end of life is regarded as the event to be modelled, one could construct probabilistic models for the time until this event, determined by a set of covariates. However, one prerequisite for establishing such modes is the availability of sufficient amount of run-to-failure data, where the time until EOL is observed for a number of batteries or bat- 
tery cells. Such data could typically be collected from similar batteries in operations to reflect realistic load profiles.

Survival analysis modelling are applied to lithium-ion batteries for end-of-performance modelling in (Y.-F. Wang, Tseng, Lindqvist, \& Tsui, 2019). A trend-renewal process is used on accelerated testing data to predict end of performance. However, this model relies on observed capacity ratios for projecting capacity fade and estimate end of performance, which will typically not be available for maritime battery systems.

\subsection{Cumulative Damage Models}

Cumulative damage models are often used for modelling of structural fatigue, where the structural deterioration is modelled as a cumulative sum of different load cycles. Fatigue life of a structure is typically given in terms of number of stress cycles of a specific amplitude. For structural components exposed to a complex, random sequence of loads, the fatigue damage can be estimated by reducing the complex loading to a series of simple cyclic loadings using techniques such as rainflow counting and then form a fatigue damage spectrum as a histogram of cyclic stresses. The degree of cumulative damage for each stress level can then be calculated from an S-N curve, that can be established based on laboratory tests. Often, simple parametric functions can be fitted to the test data to allow interpolation on the $\mathrm{S}-\mathrm{N}$ curve.

For battery cells, if one were able to construct curves or surfaces similar to $\mathrm{S}-\mathrm{N}$ curves that determines the contribution to battery degradation from individual charge/discharge cycles of specified DOD/SOC range, temperature and C-rate one could imagine that this could be used to calculate state of health based on experienced load profiles and some form of cycle counting such as rainflow counting. However, an extensive set of laboratory tests would presumably be needed, where run-to-failure tests would need to be performed for a number of different cycle amplitudes and conditions.

Cumulative damage-type modelling of battery degradation based on so-called load collectives are proposed in (Nuhic et al., 2013, 2018; You et al., 2016), and approaches based on rainflow counting are suggested in (B. Xu, Oudalov, Ulbig, Andersson, \& Kirschen, 2018; S. Li, He, Su, \& Zhao, 2020)

One potential issue with cumulative damage models is that they rely on the complete operational history of the batteries. If there are long periods with missing data, the histograms, distributions or collectives may be biased and will miss information from the period where data are missing. Hence, this puts strict requirements on the reliability of the data collection procedures and on allowable downtime. Nevertheless, for complete time histories cumulative damage models are found to perform well and may be attractive alternatives for modelling battery degradation and state of health.

\subsection{Empirical/Analytical Models}

Some methods for SOH estimation are based on fitting empirical models to various measurement data. The aim of such models is to capture relationships between battery state of health and various stress factors, such as operation time, temperature and operational loads. These models are typically based on test data and the empirical relationships can be used during operation to model state of health and capacity loss of the battery.

Analytical models proposed in the literature include numerous forms of relationships between capacity fade and number of cycles/time, such as linear (Belt, Utgikar, \& Bloom, 2011), square-root (F. Yang, Song, Dong, \& Tsui, 2019), power-law (Schmalstieg, Käbitz, Ecker, \& Sauer, 2014; Han, Ouyang, Lu, \& Li, 2014), exponential (X. Zhang, Miao, \& Liu, 2017; Tang et al., 2019; Perez et al., 2018), polynomial (Micea, Ungurean, Cârstoiu, \& Groza, 2011), sigmoid (Johnen et al., 2020) or a combination of these (Xing, Ma, Tsui, \& Pecht, 2013). More complicated models also account for differences in C-rates and temperatures ( $\mathrm{Ji}$ et al., 2020; Singh, Chen, Tan, \& Huang, 2019). Other analytical model may be based on other relationships, such as a current-time constant (J. Yang, Xia, Huang, Fu, \& Mi, 2018), moved charge (Barcellona \& Piegari, 2020), the Eyring law (Redondo-Iglesias, Venet, \& Pelissier, 2017) or a combination of several features (Kandasamy, Badrinarayanan, KAnamarlapudi, Tseng, \& Soong, 2017; Schimpe et al., 2018; Naumann, Spingler, \& Jossen, 2020; Bian et al., 2020).

The coulombic efficiency (CE) is used to establish a model for actual reversible capacity in (F. Yang et al., 2019). It is assumed that the coulombic efficiency describes the decrease in reversible capacity in successive cycles, $C_{k}=C_{k-1} C E_{k}$, where $C_{i}$ denotes the reversible capacity at cycle $i$ and $C E_{i}$ is the coulombic efficiency of cycle $i$. Then, assuming that the coulombic efficiency is constant over cycles, one arrives at the following, by iterating over cycles since the initial capacity $C_{0}: C_{k}=C_{0}\left(C E_{1} C E_{2} \cdots C E_{k}\right) \approx C_{0} C E^{k}$, see also (Arachchige, Perinpanayagam, \& Jaras, 2017). Hence, they propose the following parametric model for reversible capacity

$$
C_{k}=\alpha_{0} C E^{k}+\alpha_{1}
$$

$\alpha_{0}$ and $\alpha_{1}$ are considered model parameters, and also $C E$ is regarded as a model parameter, reflecting that it is difficult to measure $C E$ accurately. This model is compared to a simple empirical model based only on cycle number; $C_{k}=\beta_{0} \sqrt{k}+$ $\beta_{1}$, and is found to perform better.

\subsection{Other Approaches}

Some other approaches to $\mathrm{SOH}$ estimation that does not directly belong to any of the categories above have been sug- 
gested in the literature, including discrete wavelet transform (DWT) based approaches (J. Kim \& Cho, 2014; Cai, Yang, Deng, Zhao, \& Deng, 2018), geometrical approaches (Lu, Tao, \& Fan, 2014), look-up tables (Dubarry et al., 2017) and a visual cognition approach (Cheng, Tao, \& Yang, 2017).

\section{Data-Driven Models for RUL Prediction}

$\mathrm{SOH}$ estimation is an important part of battery diagnostics and can inform about the current state of the battery. RUL prediction, on the other hand projects state of health into the future in a prognostic setting and relies on some assumption on future operating conditions and loads. Hence, even though some methods for $\mathrm{SOH}$ estimation can also possibly be used for RUL prediction, different types of approaches are more specifically focusing on the prognostic part of battery health estimation. Several reviews on RUL for batteries have been presented in the literature (L. Wu et al., 2016; Su \& Chen, 2017; Y. Li et al., 2019), and approaches for RUL prediction include electrochemical models (Y. Zhang, Xiong, $\mathrm{He}, \mathrm{Qu}$, \& Pecht, 2019b), equivalent circuit models (Y. Ma, Yang, Zhou, \& Chen, 2019), empirical models (SarasketaZabala et al., 2016), particle filters (L. Chen, Wang, et al., 2020; L. Chen, An, Wang, Zhang, \& Pan, 2020), data transformations (Y. Zhang, Xiong, He, \& Pecht, 2019a; Peng et al., 2020), regression models (S. Zhang, Guo, \& Zhang, 45; F. Yang, Wang, Xu, Huang, \& Tsui, 2020; Y. Zhang, Xiong, He, \& Pecht, 2019b), ML models (W. Li, Jiao, Du, Fan, \& Zhu, 2019; L. Ren et al., 2018; H. Yang et al., 2020), timeseries models (Razavi-Far, Chakrabarti, Saif, Zio, \& Palade, 2018; Razavi-Far, Chakrabarti, Saif, \& Zio, 2019) and models based on Brownian motion with drift (D. Wang, Zhao, Yang, \& Tsui, 2017; D. Wang \& Tsui, 2018; H. Zhang, Mo, Wang, $\&$ Miao, 2020). Various health indicators are also proposed for use in prognostics of battery systems, see e.g. (Zhou, Huang, Chen, \& Tao, 2016; Sun, Hao, Pecht, \& Zhou, 2018).

Machine learning methods are used for identifying and predicting knee-points and knee-onset in capacity degradation curves in (Fermín-Cueto et al., 2020). Based on knee-point predictions, the cell's expected cycle lives are estimated and classified as short, medium or long.

\section{DISCUSSION}

\subsection{Data Availability and Requirements}

Data-driven models need training data to learn relationships between input variables and responses, and the availability of data determines both what types of models can be used and the accuracy of the model predictions. Often, training data are gathered by laboratory experiments and used to train a model that can be used in an operational setting. However, if a sufficient amount of operational data is available, it may also be possible to train models based on such data without requiring extensive laboratory testing, as suggested by e.g. (Lucu et al., 2020a, 2020b). The origin of the training data notwithstanding, available training data needs to be of sufficient quality and quantity, sufficiently representative, sufficiently complete and sufficiently relevant in order to train usable data-driven models, and the availability of such data is a crucial prerequisite for relying on data-driven models for battery capacity and state of health estimation.

Also, it is important to understand what type of operational data will be available throughout the lifetime of the battery system. It is safe to assume that data such as various current, voltage and temperature measurements will be available for the SOH algorithms, but temporal and spatial resolution may vary. Furthermore, the reliability and accuracy of derived quantities such as the state of charge will need to be assured. It needs to be determined whether the data automatically collected are sufficient, or if additional specific measurements are required, e.g. periodic tests with set load patterns and fixed conditions, or particular tests such as pulse tests and impedance or resistance measurements. From a practical point of view, it may be desirable to only rely on continuously measured data streams, but results could be improved if additional tests are carried out.

The data quality is a crucial issue for data-driven methods, and results can only be as good as the data allows. Many of the continuous variables will most likely be discretized in both time and value, and additional measurement noise will always be present. This could influence results in different degrees, and some denoising and preprocessing of the data will probably be needed. For example, for methods based on ICA/DVA relying on the differentiation of discrete signals will certainly need some type of smoothing to perform well. Hence, proper approaches to preprocessing and denoising of the data signals will need to be considered as well as the actual data-driven models.

Additional factors that may be relevant for maritime batteries have not been well studied in the literature, such as the effect of humidity, airborne salinity, vibrations and the constant movement of the ship. Such information may not be available and it should be investigated to what extent such factors influence battery degradation.

\subsection{Synthetic and Realistic Load Profiles}

Some approaches to $\mathrm{SOH}$ modelling assumes that batteries are used in a controlled way, at near-constant temperatures, with constant charge and discharge C-rates and systematically cycled within a specified voltage range. Indeed, training data obtained from laboratory tests will often be collected under such controlled situations. However, for maritime battery systems, as well as for many other applications, this is hardly the case and batteries are typically cycled only partially and under highly variable loads and environments (You et al., 2016). 
Charging is often performed with a constant current constant voltage procedure, with deterministic rather than stochastic current and voltage profiles in the different steps. Hence, methods that considers features from charging profiles may be preferred to methods relying on discharge features. However, typical charging patterns may vary and extensive use of partial fast-charging may deviate from normal charging routines under very similar conditions.

This review have seen several approaches that extracts features from partial charging curves. Hence, such features are believed to be useful and it is believed that such features can be used to estimate SOH for maritime battery systems. However, the effect of dynamically varying temperatures and currents must be taken into account also for features based on partial cycling data, and this may not be straightforward.

\subsection{Statistical and Machine Learning Models}

Some aspects to consider when selecting a statistical or a machine learning model are predictability and interpretability. Typically, more advanced machine learning models are more flexible and can accommodate complicated relationships between the input and output variables and may have higher predictive power. However, such models are often referred to as black box models in the sense that it is difficult to understand the predictions and difficult to interpret the relationship. Furthermore, complicated models may fail to generalize and are more prone to overfitting than more simplistic models.

Another aspect is how to handle uncertainty. Whereas some models provide predictive distributions, most machine learning model only give point estimates. Obviously, estimation of the uncertainty can be useful, but often comes at a computational cost. Hence, selecting a statistical or a machine learning model for $\mathrm{SOH}$ estimation is a trade-off between accuracy, generalizability, interpretability and computational cost.

One aspect of missing data is that data streams will typically not contain capacity or SOH for all data points. Hence, models that can be applied with no or limited labelled data may be needed, indicating that methods from unsupervised or semisupervised learning could be relevant (Yu et al., 2020).

\subsection{Feature Extraction and Selection}

Different modelling techniques require different types of features to explain battery degradation and different training data. For models to be useful it is also important that the selected features will be collected during operation. Hence, there is typically a need for features that can be extracted from data readily available from the battery management system, such as current, voltage and temperature measurements. From such raw data, derived features such as state of charge, number of cycles and rest time at different SOC/voltage level can also be extracted. This review has showed that there are countless approaches to extract features, sometimes referred to as health indicators, for $\mathrm{SOH}$ modelling, and which features are used to train the data-driven models may typically be more important than the actual type of statistical/ML model to employ.

\subsection{Models Based on Complete Loading History vs. Snapshot Methods}

Some of the models reviewed in this paper relies on the whole operating history of the battery cells in order to estimate $\mathrm{SOH}$, whereas others estimate $\mathrm{SOH}$ based on brief snapshots. $\mathrm{Cu}-$ mulative damage models and empirical/semi-empirical models relating $\mathrm{SOH}$ to number of cycles and other stress factors such as temperature, C-rate and SOC swing are examples of the former. Regression models on features extracted from partial charging curves or incremental capacity curves are examples of the latter. Both approaches have some advantages and disadvantages.

Cumulative damage models are attractive, since they can be used to model the accumulated degradation effect from the experienced operational profile. In essence, such models establish a relationship between the load profile or individual cycle and the change in $\mathrm{SOH}$, the $\triangle \mathrm{SOH}$. The actual $\mathrm{SOH}$ after $n$ cycles can then easily be estimated as $S O H_{n}=$ $S O H_{0}+\sum_{i=1}^{n} \Delta S O H_{i}$, where $S O H_{0}$ is the initial capacity. Moreover, if a future duty cycle can be assumed, such an $\mathrm{SOH}$ estimation model can also be used for prognostics and RUL prediction. However, one disadvantage of this approach is that the complete operational profile is needed, from the first to the current cycle. Periods of missing data will effectively render such models inaccurate. Possible remedies could be to impute values for missing data, but this is probably only possible for relatively short periods of missing data.

Methods based on regular snapshots of the data streams are very attractive in the sense that it does not require access to continuous data streams, or alternatively, accumulated data in the form of histograms or collectives representing the complete operation history. With such models, it would suffice to get batches of data at certain intervals, and if the models are able to reliably extract battery capacity and $\mathrm{SOH}$ from such snapshots, the cumulative effect since the previous batch would implicitly be estimated. Thus, if such models are found to perform well enough, they may be the preferred approach for $\mathrm{SOH}$ verification of marine battery systems.

\subsection{SOH Estimation and RUL Prediction}

Estimation of state of health $(\mathrm{SOH})$ and prediction of remaining useful life (RUL) of batteries can be considered as two sides of the same coin. SOH estimation aims at describing the current degradation state of the battery, whereas RUL predictions projects future degradation of the battery until it reaches its end of life. Hence, both depends on a method for describ- 
ing ageing as a function of various factors such as calendar time, cycle time and operating conditions related to temperature, C-rate and SOC levels. However, for RUL there is the additional need of predicting future conditions and usage patterns. For battery systems operating under variable loads, this may be challenging and some additional assumptions need to be made.

Some of the methods described above for $\mathrm{SOH}$ estimation cannot easily be adopted to predict RUL, and all methods based on direct measurements such as Coulomb counting, electrochemical impedance spectroscopy and incremental capacity analysis will be difficult to apply in a prognostics setting. However, other methods will typically be more relevant for RUL prediction than for $\mathrm{SOH}$ estimation, for example different time series models and survival models. Cumulative damage type of approaches, where degradation is modelled based on cumulative effects of the load histories, on the other hand, could presumably be adopted and used also for RUL prediction, under some assumed future loading conditions. Also, many of the empirical capacity fade models could in principle be extended to predict remaining useful life of batteries, i.e. to predict when capacity crosses a predefined threshold.

The duty cycles and operating conditions of maritime battery systems will typically be unpredictable and depend on weather and sea state conditions, loading conditions and possibly different voyage lengths and routes or different operations. However, one plausible assumption could be that past operating history is representative for the future. This approach was suggested in (Nuhic et al., 2013). However, as pointed out in (Severson et al., 2019), degradation mechanisms are typically nonlinear and degradation during earlylife cell cycles may not be strongly correlated with degradation patterns in later cycles (Harris, Harris, \& Li, 2017).

\subsection{Cell vs. Module vs. Pack level}

When establishing diagnostics methods for state of health estimation one need to consider whether to apply these on cell, module or pack (string) level and the heterogeneity of the cells within a module or a pack poses a challenge; cells within a battery system will typically not degrade uniformly. Hence, methods to identify cell differences are relevant.

$\mathrm{SOH}$ estimation at cell level could be aggregated to pack level. For example, for cells connected in series with passive equalization, the available capacity of the entire string will be determined by the capacity of the single cell with the minimum capacity, and for series-connected cells with active equalization, the available pack capacity is given by the average of the call capacities (Cordoba-Arenas, Onori, \& Rizzoni, 2015). For parallel-connected cells the available capacity will be given by the average cell capacity times the number of cells. However, earlier studies have shown that bat- tery pack lives are typically shorter than single cell life due to other degradation mechanisms (Y. Zheng, Ouyang, Lu, \& Li, 2015). Even though most studies focus on single cell data, several papers addresses $\mathrm{SOH}$ estimation of battery modules and packs, see e.g. (Diao, Jiang, Zhang, Liang, \& Pecht, 2017; C. Yang et al., 2020; Dubarry et al., 2019).

\subsection{Effect of Battery Chemistry}

Modelling approaches for a range of different battery types and chemistries have been reviewed, without a lot of emphasize on what type of batteries the various methods have been applied to. It has tacitly been assumed that the data-driven methods are agnostic to battery chemistry, in most cases, and that different chemistries can be handled by changing the model parameters or re-training models with appropriate training data. However, it should be noted that some methods may not be easily transferred to other battery chemistries, so care should be taken when selecting a modelling approach for a particular battery type. For example, it is generally known that for lithium-iron-phosphate batteries (LFP), there is a flat plateau in the SOC-OCV curve that renders voltage-based algorithms and incremental capacity analysis difficult to apply to such types of batteries (Z. Deng et al., 2016).

\subsection{Verification and Validation}

One important question for data-driven $\mathrm{SOH}$ estimation methods is to what extent they can be verified and validated to perform satisfactorily for the intended battery system. This may require a standardized platform and extensive testing data from actual degrading batteries, and the models would need to be, somehow, verified and validated particularly for each case.

It is noted that some particular methods may be prone to systematic under- or overestimation of actual capacity. For example, $\mathrm{SOH}$ estimation based on Coulomb counting of partial cycles - a technique that is utilized for maritime battery systems today - is likely to underestimate the capacity fade and thereby overestimate the actual capacity (Stroe et al., 2018). Such systematic biases for specific approaches are important to understand and account for in order to obtain reliable estimates of state of health and capacity.

\section{Summary AND CONClusion}

This paper has presented a thorough literature review of recent publications on data-driven state of health and capacity modelling of lithium-ion battery systems. More than 300 scientific papers have been reviewed and it is believed that this review gives a fair overview of current state-of-the art in datadriven SOH modelling.

Data-driven methods for $\mathrm{SOH}$ modelling can be categorized into a few groups of approaches, i.e. direct measurement 
techniques, state-space models with observers, regression type models, time-series models, survival type models, cumulative damage models and empirical/analytical models. However, the distinction is not crisp, and several types of approaches are often combined. Some of these approaches are deemed more relevant for maritime battery systems than others. One desired feature is that it only needs information contained in normal operational data. For example, time-series models require time-series of capacity measurements that will not be available, and survival type models need extensive lifetime data that cannot be expected to be available. State-space models, either electrochemical models or equivalent circuit models are typically used in BMS for SOC and SOH estimation. However, from a class perspective the aim is to develop means for independent verification of capacity/SOH estimation made by the BMS, and it may therefore be advisable to consider alternative modelling approaches. Hence, it is believed that a combination of direct measurement techniques, regression models, empirical models and cumulative damage models will be most relevant.

Direct measurement techniques include some approaches that require particular hardware and might not be suitable for online verification of SOH estimation. Moreover, direct capacity estimation based on Coulomb counting requires specific reference charge and discharge cycles, under specific conditions which will not be observed during normal operations. However, techniques based on partial charge or discharge information could be useful and will be explored further.

A large number of regression type models, ranging from simple linear regression models, to empirical/analytical models, to highly complex machine learning models have been proposed, establishing a relationship between capacity and different features extracted from the data. Perhaps more important than what type of regression model to use is the selection of features to use. Two fundamentally different approaches can be taken, herein referred to as snapshot and cumulative approaches. The cumulative approaches establish a relationship between various stress factors and capacity degradation, $\Delta C$, whereas the snapshot approach establishes a relationship between observed features and actual capacity, $C$.

One disadvantage of cumulative models is the need for the full operating history of the batteries. If parts of the history is missing it will not be possible to estimate actual capacity at a particular time. A huge advantage of snapshot models is that capacity can be estimated based on only parts of the continuous data-stream. This is believed to be a very promising feature of a method employed for regular verification of online capacity estimation. However, such methods may require higher temporal resolution in the data in order to extract the necessary features. If reliable such models can be established, SOH can be verified based on regular batches of data. However, challenges remains with respect to how the influ- ence of temperature, variations in state of charge and current can be incorporated into the models.

Notwithstanding many unresolved challenges; many of the reviewed papers explicitly state that the problem of online state-of-health estimation of lithium-ion batteries are far from being solved, this paper gives some directions for further research on data-driven estimation of battery state of health, for the purpose of verifying capacity on maritime battery systems.

\section{ACKNOWLEDGMENT}

This work has partly been carried out within the DDD BATMAN project, supported by MarTERA and the Research Council of Norway.

\section{REFERENCES}

Arachchige, B., Perinpanayagam, S., \& Jaras, R. (2017). Enhanced prognostic model for lithium ion batteries based on particle filter state transition model modification. applied sciences, 7, 1172.

Balagopal, B., \& Chow, M.-Y. (2015). The state of the art approaches to estimate the state of health $(\mathrm{SOH})$ and state of function (SOF) of lithium ion batteries. In Proceedings of the IEEE 13th international conference on industrial informatics (INDIN). IEEE.

Barai, A., Uddin, K., Dubarry, M., Sommerville, L., McGordon, A., Jennings, P., \& Bloom, I. (2019). A comparison of methodologies for non-invasive characterisation of commercial li-ion cells. Progress in Energy and Combustion Science, 72, 1-31.

Barcellona, S., \& Piegari, L. (2020). Effect of current on cycle aging of lithium ion batteries. Journal of Energy Storage, 29, 101310.

Barré, A., Deguilhem, B., Grolleau, S., \& Gérard, M. (2013). A review on lithium-ion battery ageing mechanisms and estimations for automotive applications. Journal of Power Sources, 241, 680-689.

Belt, J., Utgikar, V., \& Bloom, I. (2011). Calendar and PHEV cycle life aging of high-energy, lithium-ion cells containing blended spinel and layered-oxide cathodes. Journal of Power Sources, 196(23), 10213-10221.

Berecibar, M., Devriendt, F., Dubarry, M., Villarreal, I., Omar, N., Verbeke, W., \& Van Mierlo, J. (2016). Online state of health estimation on NMC cells based on predictive analysis. Journal of Power Sources, 320, 239-250.

Berecibar, M., Gandiaga, I., Villarreal, I., Omar, N., Van Mierlo, J., \& Van den Bossche, P. (2016). Critical review of state of health estimation methods of li-ion batteries for real applications. Renewable and Sustainable Energy Reviews, 56, 572-587.

Bi, Y., Yin, Y., \& Choe, S.-Y. (2020). Online state of 
health and aging parameter estimation using a physicsbased life model with a particle filter. Journal of Power Sources, 476, 228655:1-14.

Bian, X., Liu, L., Yan, J., Zou, Z., \& Zhao, R. (2020). An open circuit voltage-based model for state-of-health estimation of lithium-ion batteries: Model development and validation. Journal of Power Sources, 448, 227401.

Birkl, C. R., Roberts, M. R., McTurk, E., Bruce, P. G., \& Howey, D. A. (2017). Degradation diagnostics for lithium ion cells. Journal of Power Sources, 341, 373386.

Blanke, H., Bohlen, O., Buller, S., De Doncker, R. W., Fricke, B., Hammouche, A., ... Sauer, D. U. (2005). Impedance measurements on lead-acid batteries for state-of-charge, state-of-health and cranking capability prognosis in electric and hybrid electric vehicles. Journal of Power Sources, 144(2), 418-425.

Bohlen, O. S. (2008). Impedance-based battery monitoring (Tech. Rep. No. PhD thesis D 82). RWTH Aachen.

Bole, B., Kulkarni, C. S., \& Daigle, M. (2014). Adaptation of an electrochemistry-based li-ion battery model to account for deterioration observed under randomized use. In Proceedings of the annual conference of the prognostics and health management society 2014. PHM Society.

Cai, Y., Yang, L., Deng, Z., Zhao, X., \& Deng, H. (2018). Online identification of lithium-ion battery state-of-health based on fast wavelet transform and cross D-Markov machine. Energy, 147, 621-635.

Chen, L., An, J., Wang, H., Zhang, M., \& Pan, H. (2020). Remaining useful life prediction for lithium-ion battery by combining an improved particle filter with slidingwindow gray model. Energy Reports, 6, 2086-2093.

Chen, L., Lü, Z., Lin, W., Li, J., \& Pan, H. (2018). A new state-of-health estimation method for lithium-ion batteries through the intrinsic relationship between ohmic resistance and capacity. Measurement, 116, 586-595.

Chen, L., Wang, H., Jing, C., An, J., Ji, B., Lyu, Z., ... Pan, H. (2020). A novel remaining useful life prediction framework for lithium-ion battery using grey model and particle filtering. International Journal of Energy Research, 44, 7435-7449.

Chen, Z., Sun, M., Shu, X., Shen, J., \& Xiao, R. (2018). On-board state of health estimation for lithium-ion batteries based on random forest. In Proceedings of 2018 IEEE international conference on industrial technology. IEEE.

Cheng, Y., Tao, L., \& Yang, C. (2017). Lithium-ion battery capacity estimation: A method based on visual cognition. Complexity, 2017, 6342170:1-13.

Cordoba-Arenas, A., Onori, S., \& Rizzoni, G. (2015). A control-oriented lithium-ion battery pack model for plug-in hybrid electric vehicle cycle-life studies and system design with consideration of health management. Journal of Power Sources, 279, 791-808.

Cuma, M. U., \& Koroglu, T. (2015). A comprehensive review on estimation strategies used in hybrid and battery electric vehicles. Renewable and Sustainable Energy Reviews, 42, 517-531.

Daigle, M., \& Kulkarni, C. S. (2013). Electrochemistrybased battery modeling for prognostics. In Proceedings of the annual conference of the prognostics and health management society 2013. PHM Society.

Deng, Y., Ying, H., E, J., Zhu, H., Wei, K., Chen, J., . . Liao, G. (2019). Feature parameter extraction and intelligent estimation of the state-of-health of lithium-ion batteries. Energy, 176, 91-102.

Deng, Z., Yang, L., Cai, Y., \& Deng, H. (2017). Maximum available capacity and energy estimation based on support vector machine regression for lithium-ion battery. Energy Procedia, 107, 68-75.

Deng, Z., Yang, L., Cai, Y., Deng, H., \& Sun, L. (2016). Online available capacity prediction and state of charge estimation based on advanced data-driven algorithms for lithium iron phosphate battery. Energy, 112, 469480.

Diao, W., Jiang, J., Zhang, C., Liang, H., \& Pecht, M. (2017). Energy state of health estimation for battery packs based on the degradation and inconsistency. Energy Procedia, 142, 3578-3583.

DNV GL. (2016). DNV GL handbook for maritime and offshore battery systems (Tech. Rep. No. 2016-1056). Author.

DNV GL. (2020a). Rules for classification: Ships. (DNVGLRU-SHIP)

DNV GL. (2020b). Rules for classification: Ships. part 6 additional class notations. chapter 2 propulsion, power generation and auxiliary systems. (DNVGL-RU-SHIP Pt.6 Ch.2)

Doerffel, D., \& Sharkh, S. A. (2006). A critical review of using Peukert equation for determining the remaining capacity of lead-acid and lithium-ion batteries. Journal of Power Sources, 155(2), 395-400.

Dong, G., Yang, F., Wei, Z., Wei, J., \& Tsui, K. (2020). Datadriven battery health prognosis using adaptive Brownian motion model. IEEE Transaction on Industrial Informatics, 16(7), 4736-4746.

Dubarry, M., Baure, G., Pastor-Fernández, C., Yu, T. F., Widanage, W. D., \& Marco, J. (2019). Battery energy storage system modeling: A combined comprehensive approach. Journal of Energy Storage, 21, 172-185.

Dubarry, M., Berecibar, M., Devie, A., Anseán, Omar, N., \& Villarreal, I. (2017). State of health battery estimator enabling degradation diagnosis: Model and algorithm description. Journal of Power Sources, 360, 59-69.

Fan, Y., Xiao, F., Li, C., Yang, G., \& Tang, X. (2020). A novel deep learning framework for state of health estimation 
of lithium-ion battery. Journal of Energy Storage, 32, 101741.

Farmann, A., Waag, W., Marongiu, A., \& Sauer, D. U. (2015). Critical review of on-board capacity estimation techniques for lithium-ion batteries in electric and hybrid electric vehicles. Journal of Power Sources, 281, 114-130.

Feng, X., Li, J., Ouyang, M., Lu, L., Li, J., \& He, X. (2013). Using probability density function to evaluate the state of health of lithium-ion batteries. Journal of Power Sources, 232, 209-218.

Feng, X., Merla, Y., Weng, C., Ouyang, M., He, X., Liaw, B. Y., ... Offer, G. (2020). A reliable approach of differentiating discrete sampled-data for battery diagnosis. eTransportation, 3, 100051.

Fermín-Cueto, P., McTurk, E., Allerhand, M., Medina-Lopez, E., Anjos, M. F., Sylvester, J., \& dos Reis, G. (2020). Identification and machine learning prediction of kneepoint and knee-onset in capacity degradation curves of lithium-ion cells. Energy and AI, 1, 100006:1-10.

Fly, A., \& Chen, R. (2020). Rate dependency of incremental capacity analysis $(\mathrm{dQ} / \mathrm{dV})$ as a diagnostic tool for lithium-ion batteries. Journal of Energy Storage, 29, 101329.

Geisbauer, C., Wöhrl, K., Mittmann, C., \& Schweiger, H.G. (2020). Review of safety aspects of calendar aged lithium ion batteries. Journal of the Electrochemical Society, 167, 090523.

Gismero, A., Schaltz, E., \& Stroe, D.-I. (2020). Recursive state of charge and state of health estimation method for lithium-ion batteries based on coulomb counting and open circuit voltage. energies, 13, 1811.

Guo, P., Cheng, Z., \& Yang, L. (2019). A data-driven remaining capacity estimation approach for lithium-ion batteries based on charging health feature extraction. Journal of Power Sources, 412, 442-452.

Hametner, C., Jakubek, S., \& Prochazka, W. (2018). Datadriven design of a cascaded observer for battery state of health estimation. IEEE Transactions on Industry Applications, 54(6), 6258-6266.

Han, X., Feng, X., Ouyang, M., Lu, L., Li, J., Zheng, Y., \& Li, Z. (2019). A comparative study of charging voltage curve analysis and state of health estimation of lithiumion batteries in electric vehicle. Automotive Innovation, 2, 263-275.

Han, X., Lu, L., Zheng, Y., Feng, X., Li, Z., Li, J., \& Ouyang, M. (2019). A review on the key issues of the lithium ion battery degradation among the whole life cycle. eTransportation, 1, 100005.

Han, X., Ouyang, M., Lu, L., \& Li, J. (2014). A comparative study of commercial lithium ion battery cycle life in electric vehicle: Capacity loss estimation. Journal of Power Sources, 268, 658-669.

Harris, S. J., Harris, D. J., \& Li, C. (2017). Failure statis- tics for commercial lithium ion batteries: A study of 24 pouch cells. Journal of Power Sources, 342, 589-597.

Harting, N., Schenkendorf, R., Wolff, N., \& Krewer, U. (2018). State-of-health identification of lithium-ion batteries based on nonlinear frequency response analysis: First steps with machine learning. Applied Sciences, 8, 821:1-14.

Harting, N., Wolff, N., Röder, F., \& Krewer, U. (2017). Nonlinear frequency response analysis (NFRA) of lithiumion batteries. Electrochimica Acta, 248, 133-139.

He, J., Wei, Z., Bian, X., \& Yan, F. (2020). State-of-health estimation of lithium-ion batteries using incremental capacity analysis based on voltage-capacity model. IEEE Transactions on Transportation Electrification, 6(2), 417-426.

Howey, D. A., Mitcheson, P. D., Yufit, V., Offer, G. J., \& Brandon, N. P. (2014). Online measurement of battery impedance using motor controller excitation. IEEE Transactions on Vehicular Technology, 63(6), 25572566.

Huang, S.-C., Tseng, K.-H., Liang, J.-W., Chang, C.-L., \& Pecht, M. G. (2017). An online SOC and SOH estimation model for lithium-ion batteries. energies, 10(4), 512

Huixin, T., Qin, P., Li, K., \& Zhao, Z. (2020). A review of the state of health for lithium-ion batteries: Research status and suggestions. Journal of Cleaner Production, 261, 120813.

Ji, H., Zhang, W., Pan, X.-H., Hua, M., Chung, Y.-H., Shu, C.-M., \& Zhang, L.-J. (2020). State of health prediction model based on internal resistance. International Journal of Energy Research, 44, 6502-6510.

Jiang, B., Dai, H., \& Wei, X. (2020). Incremental capacity analysis based adaptive capacity estimation for lithiumion battery considering charging condition. Applied Energy, 269, 115074:1-12.

Johnen, M., Pitzen, S., Kamps, U., Kateri, M., Dechent, P., \& Sauer, D. U. (2020). Modeling long-term capacity degradation of lithium-ion batteries. Journal of Energy Storage, 34, 102011.

Kabir, M., \& Demirocak, D. E. (2017). Degradation mechanisms in li-ion batteries: a state-of-the-art review. International Journal of Energy Research, 41, 19631986.

Kandasamy, N. K., Badrinarayanan, R., KAnamarlapudi, V. R. K., Tseng, K. J., \& Soong, B.-H. (2017). Performance analysis of machine-learning approaches for modeling the charging/discharging profiles of stationary battery systems with non-uniform cell aging. batteries, $3,18$.

Karlsen, H., Dong, T., Yang, Z., \& Carvalho, R. (2019). Temperature-dependence in battery management systems for electric vehicles: Challenges, criteria and solutions. IEEE Access, 7, 142203-142213. 
Khaleghi, S., Firouz, Y., Van Mierlo, J., \& Van den Bossche, P. (2019). Developing a real-time data-driven battery health diagnosis method, using time and frequency domain condition indicators. Applied Energy, $255,113813$.

Khumprom, P., \& Yodo, N. (2019). A data-driven predictive prognostic model for lithium-ion batteries based on a deep learning algorithm. energies, 12(4), 660.

Kim, J., \& Cho, B. (2014). An innovative approach for characteristic analysis and state-of-health diagnosis for a liion cell based on the discrete wavelet transform. Journal of Power sources, 260, 115-130.

Kim, T., Wang, Y., Sahinoglu, Z., Wada, T., Hara, S., \& Qiao, W. (2015). A Rayleigh quotient-based recursive totalleast-squares online maximum capacity estimation for lithium-ion batteries. IEEE Transactions on Energy Conversion, 30(3), 842-851.

Klass, V., Behm, M., \& Lindberg, G. (2014). A support vector machine-based state-of-health estimation method for lithium-ion batteries under electric vehicle operation. Journal of Power Sources, 270, 262-272.

Kuipers, M., Schröer, P., Nemeth, T., Zappen, H., Blömeke, \& Sauer, D. U. (2020). An algorithm for an online electrochemical impedance spectroscopy and battery parameter estimation: Development, verification and validation. Journal of Energy Storage, 30, 101517.

Kwon, S.-J., Han, D., Choi, J. H., Lim, J.-H., Lee, S.E., \& Kim, J. (2020). Remaining-useful-life prediction via multiple linear regression and recurrent neural network reflecting degradation information of a $20 \mathrm{Ah}$ $\mathrm{LiNi}_{x} \mathrm{Mn}_{y} \mathrm{Co}_{1-x-y} \mathrm{O}_{2}$ pouch cell. Journal of Electroanalytical Chemistry, 858, 113729.

Le, D., \& Tang, X. (2011). Lithium-ion battery state of health estimation using Ah-V characterization. In Proceedings of the annual conference of the prognostics and health management society 2011. PHM Society.

Lemprecht, A., Riesterer, M., \& Steinhorst, S. (2020). Random forest regression of charge balancing data: A state of health estimation method for electric vehicle batteries. In Proceedings of 2020 international conference on omni-layer intelligent systems. IEEE.

Ley, C. P., \& Orchard, M. E. (2021). Simultaneous inference of lithium ion battery polarising impedance surface and capacity degradation using a hybrid neural adaptive state space model. Journal of Energy Storage, 36, 102370.

Li, L., Wang, P., Chao, K.-H., Zhou, Y., \& Xie, Y. (2016). Remaining useful life prediction for lithium-ion batteries based on Gaussian processes mixture. PLOS ONE, 11(9), 1-13.

Li, P., Zhang, Z., Xiong, Q., Ding, B., Hou, J., Luo, D., ... Li, S. (2020). State-of-health estimation and remaining useful life prediction for the lithium-ion battery based on a variant long short term memory neural network.
Journal of Power Sources, 459, 228069.

Li, S., He, H., Su, C., \& Zhao, P. (2020). Data driven battery modeling and management method with aging phenomenon considered. Applied Energy, 275, 115340.

Li, W., Jiao, Z., Du, L., Fan, W., \& Zhu, Y. (2019). An indirect RUL prognosis for lithium-ion battery under vibration stress using Elman neural network. International Journal of Hydrogen Energy, 44, 12270-12276.

Li, W., Sengupta, N., Dechent, P., Howey, D., Annaswamy, A., \& Sauer, D. U. (2021). Online capacity estimation of lithium-ion batteries with deep long short-term memory networks. Journal of Power Sources, 482, 228863:1-11.

Li, X., Wang, Z., \& Yan, J. (2019). Prognostic health condition for lithium battery using the partial incremental capacity and Gaussian process regression. Journal of Power Sources, 421, 56-67.

Li, X., Wang, Z., Zhang, L., Zou, C., \& Dorell, D. D. (2019). State-of-health estimation of li-ion batteries by combining the incremental capacity analysis method with grey relational analysis. Journal of Power Sources, 410-411, 106-114.

Li, X., Yuan, C., \& Wang, Z. (2020a). Multi-time-scale framework for prognostic health condition of lithium battery using modified Gaussian process regression and nonlinear regression. Journal of Power Sources, 467, 228358.

Li, X., Yuan, C., \& Wang, Z. (2020b). State of health estimation for li-ion battery via partial incremental capacity analysis based on support vector regression. Energy, 203, 117852.

Li, X., Zhang, L., Wang, Z., \& Dong, P. (19). Remaining useful life prediction for lithium-ion batteries based on a hybrid model combining the long short-term memory and Elman neural networks. Journal of Energy Storage, 21, 510-518.

Li, Y., Abdel-Monem, M., Gopalakrishnan, R., Berecibar, M., Nanini-Maury, E., Omar, N., ... Van Mierlo, J. (2018). A quick on-line state of health estimation method for li-ion battery with incremental capacity curves processed by Gaussian filter. Journal of Power Sources, 373, 40-53.

Li, Y., Liu, K., Foley, A. M., Zülke, A., Berecibar, M., Nanini-Maury, E., ... Hoster, H. E. (2019). Datadriven health estimation and lifetime prediction of lithium-ion batteries: A review. Renewable and Sustainable Energy Reviews, 113, 109254.

Li, Y., Sheng, H., Cheng, Y., Stroe, D.-I., \& Teodorescu, R. (2020). State-of-health estimation of lithium-ion batteries based on semi-supervised transfer component analysis. Applied Energy, 277, 115504.

Li, Y., Zou, C., Berecibar, M., Nanini-Maury, E., Chan, J. C.W., van den Bossche, P., ... Omar, N. (2018). Random forest regression for online capacity estimation of 
lithium-ion batteries. Applied Energy, 232, 197-210.

Lin, C., Cabrera, J., Yu, Y., Denis, Yang, F., \& Tsui, K. (2020). SOH estimation and SOC recalibration of lithium-ion battery with incremental capacity analysis \& cubic smoothing spline. Journal of The Electrochemical Society, 167, 090537.

Lin, C., Xing, J., \& Tang, A. (2017). Litium-ion battery state of charge/state of health estimation using SMO for EVs. Energy Procedia, 105, 4383-4388.

Lin, C.-P., Cabrera, J., Yang, F., Ling, M.-H., Tsui, K.-L., \& Bae, S.-J. (2020). Battery state of health modeling and remaining useful life prediction through time series model. Applied Energy, 275, 115338:1-21.

Lin, H.-T., Liang, T.-J., \& Chen, S.-M. (2013). Estimation of battery state of health using probabilistic neural network. IEEE Transactions on Industrial Informatics, 9(2), 679-685.

Lipu, M. H., Hannan, M., Hussain, A., Hoque, M., Ker, P. J., Saad, M., \& Ayob, A. (2018). A review of state of health and remaining useful life estimation methods for lithium-ion battery in electric vehicles: Challenges and recommendations. Journal of Cleaner Production, 205, 115-133.

Liu, D., Luo, Y., Liu, J., Peng, Y., Guo, L., \& Pecht, M. (2014). Lithium-ion battery remaining useful life estimation based on fusion nonlinear degradation AR model and RPF algorithm. Neural Computing and Applications, 25, 557-572.

Lu, C., Tao, L., \& Fan, H. (2014). Li-ion battery capacity estimation: A geometrical approach. Journal of Power Sources, 261, 141-147.

Lucu, M., Martinez-Laserna, E., Gandiaga, I., \& Camblong, H. (2018). A critical review of self-adaptive li-ion battery ageing models. Journal of Power Sources, 401, 85-101.

Lucu, M., Martinez-Laserna, E., Gandiaga, I., Liu, K., Camblong, H., Widanage, W., \& Marco, J. (2020a). Datadriven nonparametric li-ion battery ageing model aiming at learning from real operation data - part a: Storage operation. Journal of Energy Storage, 30, 101409.

Lucu, M., Martinez-Laserna, E., Gandiaga, I., Liu, K., Camblong, H., Widanage, W., \& Marco, J. (2020b). Data-driven nonparametric li-ion battery ageing model aiming at learning from real operation data - part b: Cycling operation. Journal of Energy Storage, 30, 101410.

Ma, G., Zhang, Y., Cheng, C., Zhou, B., Hu, P., \& Yuan, Y. (2019). Remaining useful life prediction of lithium-ion batteries based on false nearest neighbors and a hybrid neural network. Applied Energy, 253, 113626.

Ma, Y., Wu, L., Guan, Y., \& Peng, Z. (2020). The capacity estimation and cycle life prediction of lithium-ion batteries using a new broad extreme learning machine approach. Journal of Power Sources, 476, 228581:1-
11.

Ma, Y., Yang, C., Zhou, X., \& Chen, H. (2019). Remaining useful life prediction of lithium-ion battery based on Gauss-Hermite particle filter. IEEE Transactions on Control Systems Technology, 27(4), 1788-1795.

Meng, J., Cai, L., Stroe, D.-I., Luo, G., Sui, X., \& Teodorescu, R. (2019). Lithium-ion battery state-of-health estimation in electric vehicle using optimized partial charging voltage profiles. Energy, 185, 1054-1062.

Micea, M. V., Ungurean, L., Cârstoiu, G. N., \& Groza, V. (2011). Online state-of-health assessment for battery management systems. IEEE Transactions on Instrumentation and Measurement, 60(6), 1997-2006.

Murashko, K., Mityakov, A., Mityakov, V., Sapozhnikov, S., Pyrhönen, J., \& Jokiniemi, J. (2019). Cylindrical li-ion battery state of health evaluation by differential heat analysis during calendar ageing. Journal of the Electrochemical Society, 166(13), A2896-A2902.

Naha, A., Han, S., Agarwal, s., Guha, A., Khandelwal, A., Tagade, P., ... Oh, B. (2020). An incremental voltage difference based technique for online state of health estimation of li-ion batteries. Scientific Reports, 10, 9526:1-11.

Naumann, M., Spingler, F. B., \& Jossen, A. (2020). Analysis and modeling of cycle aging of a commercial LiFePO4/graphite cell. Journal of Power Sources, 451, 227666.

Nuhic, A., Bergdolt, J., Spier, B., Buchholz, M., \& Dietmayer, K. (2018). Battery heath monitoring and degradation prognosis in fleet management systems. World Electric Vehicle Journal, 9(3), 39:1-20.

Nuhic, A., Terzmehic, T., Soczka-Guth, T., Buchholz, M., \& Dietmayer, K. (2013). Health diagnosis and remaining useful life prognostics of lithium-ion batteries using data-driving methods. Journal of Power Sources, 239, 680-688.

Pan, H., Lü, Z., Wang, H., Wei, H., \& Chen, L. (2018). Novel battery state-of-health online estimation method using multiple health indicators and an extreme learning machine. Energy, 160, 466-477.

Pan, W., Chen, Q., Zhu, M., Tang, J., \& Wang, J. (2020). A data-driven fuzzy information granulation approach for battery state of health forecasting. Journal of Power Sources, 475, 228716:1-9.

Pastor-Fernández, C., Yu, T. F., Widanage, W. D., \& Marco, J. (2019). Critical review of non-invasive diagnosis techniques for quantification of degradation modes in lithium-ion batteries. Renewable and Sustainable Energy Reviews, 109, 138-159.

Pelletier, S., Jabali, O., Laporte, G., \& Veneroni, M. (2017). Battery degradation and behaviour for electric vehicles: Review and numerical analysis of several models. Transportation Research Part B, 103, 158-187.

Peng, J., Zheng, Z., Zhang, X., Deng, K., Gao, K., Li, H., ... 
Huang, Z. (2020). A data-driven method with feature enhancement and adaptive optimization for lithium-ion battery remaining useful life prediction. energies, 13 , 752.

Pérez, A., Benavides, M., Rozas, H., Seria, S., \& Orchard, M. (2018). Guidelines for the characterization of the internal impedance of lithium-ion batteries in PHM algorithms. International Journal of Prognostics and Health Management, 9(3), 1-10.

Perez, A., Quintero, V., Jaramillo, F., Rozas, H., Jimenez, D., Orchard, M., \& Moreno, R. (2018). Characterization of the degradation process of lithium-ion batteries when discharged at different current rates. Proceedings of the Institution of Mechanical Engineers, Part I: Journal of Systems and Control Engineering, 232(8), 1075-1089.

Plett, G. L. (2004a). Extended Kalman filtering for battery management systems of LiPB-based HEV battery packs. part 1. background. Journal of Power Sources, 134, 252-261.

Plett, G. L. (2004b). Extended Kalman filtering for battery management systems of LiPB-based HEV battery packs. part 2. modeling and identification. Journal of Power Sources, 134, 262-276.

Plett, G. L. (2004c). Extended Kalman filtering for battery management systems of LiPB-based HEV battery packs. part 3. state and parameter estimation. Journal of Power Sources, 134, 277-292.

Plett, G. L. (2011). Recursive approximate weighted total least squares estimation of battery cell total capacity. Journal of Power Sources, 196, 2319-2331.

Razavi-Far, R., Chakrabarti, S., Saif, M., \& Zio, E. (2019). An integrated imputation-prediction scheme for prognostics of battery data with missing observations. Expert Systems With Applications, 115, 709-723.

Razavi-Far, R., Chakrabarti, S., Saif, M., Zio, E., \& Palade, V. (2018). Extreme learning machine based prognostics of battery life. International Journal of Artificial Intelligence Tools, 27(08), 1850036.

Redondo-Iglesias, E., Venet, P., \& Pelissier, s. (2017). Eyring acceleration model for predicting calendar ageing of lithium-ion batteries. Journal of Energy Storage, 13, 176-183.

Ren, D., Hsu, H., Li, R., Feng, X., Guo, D., Han, X., ... Ouyang, M. (2019). A comparative investigation of aging effects on thermal runaway behavior of lithiumion batteries. eTransportation, 2, 100034.

Ren, L., Zhao, L., Hong, S., Zhao, S., Wang, H., \& Zhang, L. (2018). Remaining useful life prediction for lithiumion battery: A deep learning approach. IEEE Access, 6, 50587-50598.

Richardson, R. R., Birkl, C. R., Osborne, M. A., \& Howey, D. A. (2019). Gaussian process regression for in situ capacity estimation of lithium-ion batteries. IEEE Transactions on Industrial Informatics, 15(1), 127-
138.

Richardson, R. R., Osborne, M. A., \& Howey, D. A. (2019). Battery health prediction under generalized conditions using a Gaussian process transition model. Journal of Energy Storage, 23, 320-328.

Riviere, E., Sari, A., Venet, P., Meniere, F., \& Bultel, Y. (2019). Innovative incremental capacity analysis implementation for $\mathrm{C} / \mathrm{LiFePO}_{4}$ cell state-of-health estimation in electrical vehicles. Batteries, 5(2), 37:13.

Rozas, H., Troncoso-Kurtovic, D., Ley, C. P., \& Orchard, M. E. (2021). Lithium-ion battery state-of-latentenergy (SoLE): A fresh new look to the problem of energy autonomy prognostics in storage systems. Journal of Energy Storage, 40, 102735.

Saha, B., Goebel, K., \& Chrisophersen, J. (2009). Comparison of prognostic algorithms for estimating remaining useful life of batteries. Transactions of the Institute of Measurement and Control, 31(3/4), 293-308.

Saqli, K., Bouchareb, H., Oudghiri, M., \& M'Sirdi, N. (2020). Critical review of ageing mechanisms and state of health estimation methods for battery performance. In J. Littlewood, R. J. Howlett, A. Capozzoli, \& L. C. Jain (Eds.), Sustainability in energy and buildings (Vol. 163, p. 507-518). Singapore: Springer Nature.

Sarasketa-Zabala, E., Martinez-Laserna, E., Berecibar, M., Gandiaga, I., Rodriguez-Martinez, L., \& Villarreal, I. (2016). Realistic lifetime prediction approach for $\mathrm{Li}$ ion batteries. Applied Energy, 162, 839-852.

Sbarufatti, C., Corbetta, M., Giglio, M., \& Cadini, F. (2017). Adaptive prognosis of lithium-ion batteries based on the combination of particle filters and radial basis function neural networks. Journal of Power Sources, 344, 128-140.

Schimpe, M., von Kuepach, M., Naumann, M., Hesse, H., Smith, K., \& Jossen, A. (2018). Comprehensive modeling of temperature-dependent degradation mechanisms in lithium iron phosphate batteries. Journal of The Electrochemical Society, 165(2), A181-A193.

Schmalstieg, J., Käbitz, S., Ecker, M., \& Sauer, D. U. (2014). A holistic aging model for $\mathrm{Li}(\mathrm{NiMnCo}) \mathrm{O}_{2}$ based 18650 lithiium-ion batteries. Journal of Power Sources, 257, 325-334.

Severson, K. A., Attia, P. M., Jin, N., Perkins, N., Jiang, B., Yang, Z., ... Braats, R. D. (2019). Data-driven prediction of battery cycle life before capacity degradation. Nature Energy, 4, 383-391.

Shen, D., Wu, L., Kang, G., Guan, Y., \& Peng, Z. (2021). A novel online method for predicting the remaining useful life of lithium-ion batteries considering rendom variable discharge current. Energy, 218, 119490:1-17.

Shen, S., Sadoughi, M., Chen, X., Hong, M., \& Hu, C. (2019). A deep learning method for online capacity estimation of lithium-ion batteries. Journal of Energy Storage, 25, 100817. 
Shen, S., Sadoughi, M., Li, M., Wang, Z., \& Hu, C. (2020). Deep convolutional neural networks with ensemble learning and transfer learning for capacity estimation of lithium-ion batteries. Applied Energy, 260, 114296.

Shu, X., Li, G., Shen, J., Lei, Z., Chen, Z., \& Liu, Y. (2020). A uniform estimation framework for the state of health of lithium-ion batteries considering feature extraction and parameters optimization. Energy, 204, 117957.

Shu, X., Li, G., Zhang, Y., Shen, J., Chen, Z., \& Liu, Y. (2020). Online diagnosis of state of health for lithium-ion batteries based on short-term charging profiles. Journal of Power Sources, 471, 228478.

Singh, P., Chen, C., Tan, C. M., \& Huang, S.-C. (2019). Semiempirical capacity fading model for SoH estimation of li-ion batteries. Applied Sciences, 9, 3012:1-15.

Song, S., Fei, C., \& Xia, H. (2020). Lithium-ion battery SOH estimation based on XGBoost algorithm with accuracy correction. Energies, 13, 812:1-13.

Stiaszny, B., Ziegler, J. C., Krauß, E. E., Schmidt, J. P., \& Ivers-Tiffée, E. (2014). Electrochemical characterization and post-mortem analysis of aged $\mathrm{LiMn}_{2} \mathrm{O}_{4}{ }^{-}$ $\mathrm{Li}\left(\mathrm{Ni}_{0.5} \mathrm{Mn}_{0.3} \mathrm{Co}_{0.2}\right) \mathrm{O}_{2} /$ graphite lithium ion batteries. part I: Cycle aging. Journal of Power Sources, 251, 439-450.

Stiaszny, B., Ziegler, J. C., Krauß, E. E., Zhang, M., \& IversTiffée, E. (2014). Electrochemical characterization and post-mortem analysis of aged $\mathrm{LiMn}_{2} \mathrm{O}_{4}-\mathrm{NMC} /$ graphite lithium ion batteries. part II: Calendar aging. Journal of Power Sources, 258, 61-75.

Stroe, D.-I., Knap, V., \& Schaltz, E. (2018). State-of-health estimation of lithium-ion batteries based on partial charging voltage profiles. ECS Transactions, 85(13), 379-386.

Su, C., \& Chen, H. (2017). A review on prognostics approaches for remaining useful life of lithium-ion battery. IOP Conference Series: EArth and Environmental Science, 93, 012040:1-8.

Sun, Y., Hao, X., Pecht, M., \& Zhou, Y. (2018). Remaining useful life prediction for lithium-ion batteries based on an integrated health indicator. Microelectronics Reliability, 88-90, 1189-1194.

Tang, X., Zou, C., Yao, K., Chen, G., Liu, B., He, Z., \& Gao, F. (2018). A fast estimation algorithm for lithium-ion state of health. Journal of Power Sources, 396, 453458.

Tang, X., Zou, C., Yao, K., Lu, J., Xia, Y., \& Gao, F. (2019). Aging trajectory prediction for lithium-ion batteries via model migration and Bayesian Monte Carlo method. Applied Energy, 254, 113591.

Tian, N., Wang, Y., Chen, J., \& Fang, H. (2020). On-shot parameter identification of the Thevenin's model for batteries: Methods and validation. Journal of Energy Storage, 29, 101282:1-11.

Tröltzsch, U., Kanoun, O., \& Tränkler, H.-R. (2006). Char- acterizing aging effects of lithium ion batteries by impedance spectroscopy. Electrochimica Acta, 51(89), 1664-1672.

Tseng, K.-H., Liang, J.-W., Chang, W., \& Huang, S.-C. (2015). Regression models using fully discharged voltage and internal resistance for state of health estimation of lithium-ion batteries. Energies, 8, 2889-2907.

Ungurean, L., Cârstoiu, G., Micea, M. V., \& Groza, V. (2017). Battery state of health estimation: a structured review of models, methods and commercial devices. International Journal of Energy Research, 41, 151-181.

Ungurean, L., Micea, M. V., \& Cârstoiu, G. (2020). Online state of health prediction method for lithium-ion batteries, based on gated recurrent unit neural networks. International Journal of Energy Research, 44(8), 67676777.

Vanem, E., Bertinelli Salucci, C., Bakdi, A., \& Alnes, Ø. A. (2021). Data-driven state of health modelling - a review of state of the art and reflections on applications for maritime battery systems. Journal of Energy Storage, 43, 103158.

Vetter, J., Novák, P., Wagner, M., Veit, C., Möller, K.-C., Besenhard, J., ... Hammouche, A. (2005). Ageing mechanisms in lithium-ion batteries. Journal of Power Sources, 147, 269-281.

Wang, D., Kong, J.-z., Yang, F., Zhao, Y., \& Tsui, K.-L. (2020). Battery prognostics at different operating conditions. Measurement, 151, 107182.

Wang, D., \& Tsui, K.-L. (2018). Brownian motion with adaptive drift for remaining useful life prediction: Revisited. Mechanical Systems and Signal Processing, 99, 691-701.

Wang, D., Zhao, Y., Yang, F., \& Tsui, K.-L. (2017). Ninlinear-drifted Brownian motion with multiple hidden states for remaining useful life prediction of rechargeable batteries. Mechanical Systems and Signal Processing, 93, 531-544.

Wang, X., Wei, X., \& Dai, H. (2019). Estimation of state of health of lithium-ion batteries based on charge transfer resistance considering different temperature and state of charge. Journal of Energy Storage, 21, 618-631.

Wang, Y.-F., Tseng, S.-T., Lindqvist, B. H., \& Tsui, K.-L. (2019). End of performance prediction of lithium-ion batteries. Journal of Quality Technology, 51(2), 198213.

Wang, Z., Zeng, S., Guo, J., \& Qin, T. (2018). Remaining capacity estimation of lithium-ion batteries based on the constant voltage charging profile. PLOS ONE, 13(7), $1-22$.

Weicker, P. (2014). A systems approach to lithium-ion battery management. Artech House.

Weng, C., Cui, Y., Sun, J., \& Peng, H. (2013). On-board state of health monitoring of lithium-ion batteries using incremental capacity analysis with support vector 
regression. Journal of Power Sources, 36-44.

Weng, C., Sun, J., \& Peng, H. (2013). An open-circuitvoltage model of lithium-ion batteries for effective incremental capacity analysis. In Proceedings of the asme 2013 dynamic systems and control conference (dscc2013). American Society of Mechanical Engineers (ASME).

Wu, B., Yufit, V., Merla, Y., Martinez-Botas, R. f., Brandon, N. P., \& Offer, G. J. (2015). Differential thermal voltammetry for tracking degradation in lithiumion batteries. Journal of Power Sources, 273, 495-501.

Wu, L., Fu, X., \& Guan, Y. (2016). Review of the remaining useful life prognostics of vehicle lithium-ion batteries using data-driven methodologies. applied sciences, 6 , 166.

Xing, Y., Ma, E. W., Tsui, K.-L., \& Pecht, M. (2013). An ensemble model for predicting the remaining useful performance of lithium-ion batteries. Microelectronics Reliability, 53, 811-820.

Xiong, R., Li, L., \& Tian, J. (2018). Towards a smarter battery management system: A critical review on battery state of health monitoring methods. Journal of Power Sources, 405, 18-29.

Xu, B., Oudalov, A., Ulbig, A., Andersson, G., \& Kirschen, D. S. (2018). Modeling of lithium-ion battery degradation for cell life assessment. IEEE Transactions on Smart Grid, 9(2), 1131-1140.

Xu, H., Peng, Y., \& Su, L. (2018). Health state estimation method of lithium ion battery based on NASA experimental data set. IOP Conference Series: Materials Science and Engineering, 452, 032067:1-7.

Xu, T., Peng, Z., \& Wu, L. (2021). A novel datadriven method for predicting the circulating capacity of lithium-ion battery under random variable current. Energy, 218, 119530:1-13.

Xu, X., Yu, C., Tang, S., Sun, X., Si, X., \& Wu, L. (2019). State-of-health estimation for lithium-ion batteries based on Wiener process with modelling the relaxation effect. IEEE Access, 7, 105186-105201.

Yang, C., Wang, X., Fang, Q., Dai, H., Cao, Y., \& Wei, X. (2020). An online SOC and capacity estimation method for aged lithium-ion battery pack considering cell inconsistency. Journal of Energy Storage, 29, 101250.

Yang, D., Wang, Y., Pan, R., Chen, R., \& Chen, Z. (2018). State-of-health estimation for the lithium-ion battery based on support vector regression. Applied Energy, 227, 273-283.

Yang, F., Song, X., Dong, G., \& Tsui, K.-L. (2019). A coulombic efficiency-based model for prognostics and health estimation of lithium-ion batteries. Energy, 171, 1173-1182.

Yang, F., Wang, D., Xu, F., Huang, Z., \& Tsui, K.-L. (2020). Lifespan prediction of lithium-ion batteries based on various extracted features and gradient boosting regression tree model. Journal of Power Sources, 476, 228654:1-10.

Yang, H., Wang, P., An, Y., Shi, C., Sun, X., Wang, K., ... Ma, Y. (2020). Remaining useful life prediction based on denoising technique and deep neural network for lithium-ion capacitors. eTransportation, 5, 100078:111.

Yang, J., Xia, B., Huang, W., Fu, Y., \& Mi, C. (2018). Online state-of-health estimation for lithium-ion batteries using constant-voltage charging current analysis. Applied Energy, 212, 1589-1600.

Yang, Q., Xu, J., Cao, B., Xu, D., Li, X., \& Wang, b. (2017). State-of-health estimation of lithium-ion battery based on interval capacity. Energy Procedia, 105, 23422347.

You, G.-w., Park, S., \& Oh, D. (2016). Real-time state-ofhealth estimation for electric vehicle batteries: A datadriven approach. Applied Energy, 176, 92-103.

Yu, J., Yang, J., Wu, Y., Tang, D., \& Dai, J. (2020). Online state-of-health prediction of lithium-ion batteries with limited labeled data. International Journal of Energy Research, 44(14), 11345-11360.

Zhang, H., Mo, Z., Wang, J., \& Miao, Q. (2020). Nonlineardrifter fractional Brownian motion with multiple hidden state variables for remaining useful life prediction of lithium-ion batteries. IEEE Transactions on Reliability, 69(2), 768-780.

Zhang, S., Guo, X., Dou, X., \& Zhang, X. (2020). A rapid online calculation method for state of health of lithiumion battery based on coulomb counting method and differential voltage analysis. Journal of Power Sources, 479, 228740:1-11.

Zhang, S., Guo, X., \& Zhang, X. (45). Multi-objective decision analysis for data-driven based estimation of battery states: A case study of remaining useful life estimation. International Journal of Hydrogen Energy, 2020, 14156-14173.

Zhang, S., Zhai, B., Guo, X., Wang, K., Peng, N., \& Zhang, X. (2019). Synchronous estimation of state of health and remaining useful lifetime for lithium-ion battery using the incremental capacity and artificial neural networks. Journal of Energy Storage, 26, 100951.

Zhang, X., Miao, Q., \& Liu, Z. (2017). Remaining useful life prediction of lithium-ion battery using an improved UPF method based on MCMC. Microelectronics Reliability, 75, 288-295.

Zhang, Y., Tang, Q., Zhang, Y., Wang, J., Stimming, U., \& Lee, A. A. (2020). Identifying degradation patterns of lithium ion batteries from impedance spectroscopy using machine learning. nature communications, 11 , 1706:1-6.

Zhang, Y., Xiong, R., He, H., \& Pecht, M. (2019b). Validation and verification of a hybrid method for remaining 
useful life prediction of lithium-ion batteries. Journal of Cleaner Production, 212, 240-249.

Zhang, Y., Xiong, R., He, H., \& Pecht, M. G. (2019a). Lithium-ion battery remaining useful life prediction with Box-Cox transformation and Monte Carlo simulation. IEEE Transactions on Industrial Electronics, 66(2), 1585-1597.

Zhang, Y., Xiong, R., He, H., Qu, X., \& Pecht, M. (2019a). Aging characteristics-based health diagnosis and remaining useful life prognostics for lithium-ion batteries. eTransportation, 1, 100004.

Zhang, Y., Xiong, R., He, H., Qu, X., \& Pecht, M. (2019b). State of charge-dependent aging mechanisms in graphite/ $\mathrm{Li}(\mathrm{NiCoAl}) \mathrm{O}_{2}$ cells: Capacity loss modelling and remaining useful life prediction. Applied Energy, 255, 113818.

Zhao, Q., Qin, X., Zhao, H., \& Feng, W. (2018). A novel prediction method based on the support vector regression for the remaining useful life of lithium-ion batteries. Microelectronics Reliability, 85, 99-108.

Zheng, L., Zhu, J., Lu, D. D.-C., Wang, G., \& He, T. (2018). Incremental capacity analysis and differential voltage analysis based state of charge and capacity estimation for lithium-ion batteries. Energy, 150, 759-769.

Zheng, Y., Ouyang, M., Lu, L., \& Li, J. (2015). Understanding aging mechanisms in lithium-ion battery packs: From cell capacity loss to pack capacity evolution. Journal of Power Sources, 278, 287-295.

Zhou, Y., \& Huang, M. (2016). Lithium-ion batteries remaining useful life prediction based on a mixture of empirical mode decomposition and ARIMA model. Microelectronics Reliability, 65, 265-273.

Zhou, Y., Huang, M., Chen, Y., \& Tao, Y. (2016). A novel health indicator for on-line lithium-ion batteries remaining useful life prediction. Journal of Power Sources, 321, 1-10. 